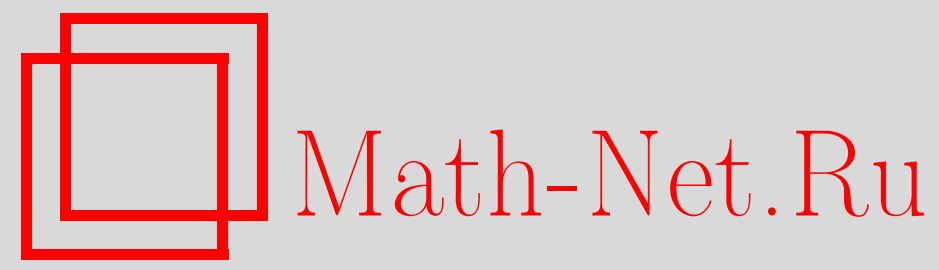

M. Minozzo, Purely game-theoretic random sequences: II. Limiting empirical distributions and strong central limit theorem, Теория вероятн. и ее примен., 2000, том 45, выпуск 2, 312-327

DOI: https://doi.org/10.4213/tvp465

Использование Общероссийского математического портала Math-Net.Ru подразумевает, что вы прочитали и согласны с пользовательским соглашением http://www . mathnet.ru/rus/agreement

Параметры загрузки:

IP : 3.85 .5 .30

26 апреля 2023 г., 13:50:14

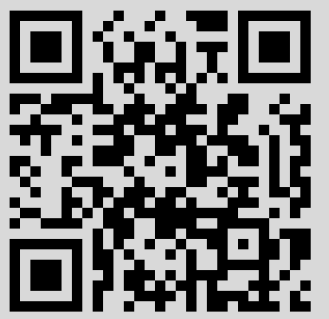




\title{
PURELY GAME-THEORETIC RANDOM SEQUENCES: II. LIMITING EMPIRICAL DISTRIBUTIONS AND STRONG CENTRAL LIMIT THEOREM
}

\begin{abstract}
В предыдущей работе автора [5] дано определение типических последовательностей, при этом не использовалось никакое колмогоровское вероятностное распределение, а непосредственно к измеримым функциям применялся принцип исключений игровой стратегии. В данной статье мы развиваем эту теорию, выводя для этих типических последовательностей некоторые элементарные предельные эмпирические функции распределения и доказывая результаты типа сильной центральной предельной теоремы для процесса бросания монеты.
\end{abstract}

Ключевые слова и фраэы: алгоритмическая теория вероятностей, предельные (в смысле сходимости п.н.) теоремы, мартингалы, типические последовательности.

\section{Introduction}

This paper is the second of two papers on a purely game-theoretic algorithmic approach to the definition of random sequences. Whereas in [5] the author introduced the basic framework and derived versions of Kolmogorov's strong law of large numbers (SLLN), and of the upper half of Kolmogorov's law of the iterated logarithm (LIL), in this paper we tackle the problem of deriving the distributions of the values, of the outcomes corresponding to a single $M$-typical sequence, for some stochastic sequences obtained from a basic martingale $M$.

Whereas in the traditional algorithmic probability frameworks, assuming a probability distribution $\mathbf{P}$, direct algorithmic versions of classical almost sure limit theorems like the SLLN or the LIL, have been obtained, there is a lack of direct algorithmic results about the statistical distributions of the outcomes corresponding to a single random sequence, and the relevant literature contains only a few examples about some relative frequency regularities. (To give one example, the most famous of these regularities, with respect to the uniform Bernoulli measure, is called, after Emile Borel,

\footnotetext{
${ }^{*}$ University of Perugia, Department of Statistical Sciences, Via A. Pascoli, 06100 Perugia, Italy; e-mail: minozzo@stat.unipg.it
} 
«normality». He called an infinite sequence of zeros and ones normal if, for each $q \in \mathbf{N}$, the frequency of occurrences of each block of digits of length $q$ in the initial segment of length $N$ tends to the limit $2^{-q}$ for $N$ going to infinity.)

Now, as far as we are concerned in this paper, in Section 2, we will consider the distributions of the values, or, using a more common terminology, the limiting empirical distributions, of the outcomes corresponding to a single $M$-typical sequence, of a Bernoulli, a moving average, and a first-order autoregressive stochastic sequence respectively. From the point of view of applications, the cases considered are fairly elementary and the basic martingale $M$ will represent essentially a coin-tossing process. These results will provide a purely game-theoretic algorithmic analogue of the strong consistency, in Kolmogorov's probability axiomatics, of the empirical distribution functions of the corresponding stochastic processes. On this line of results, in Sections 3 and 4 we will then consider some strong central limit theorem (SCLT) type results, which concern the distributions of the values, in logarithmic density, or in suitable subsequences, of the outcomes corresponding to a single $M$-typical sequence, of a standardized sum statistic.

The notation is the same as in [5], with the following additions. The indicator function of the interval $(-\infty, z], z \in(-\infty, \infty)$, is denoted by $I_{(-\infty, z]}(\cdot)$. And the cardinality of a set $A$, that is, the number of elements it contains, is denoted as $\# A$. We denote the set of all computable real numbers by $\mathbf{R}_{c}$.

\section{Limiting empirical distributions}

Throughout this section we always consider a univariate binary basic martingale difference sequence with only two possible fixed values, for example $\{-1,1\}$. If the values of the univariate binary basic martingale difference sequence were not fixed (as in the case of the upper half of the LIL of [5, Section 5]) we could not guarantee, in general, the existence of a distribution of the values for any given $M$-typical sequence. For instance, no sequence, $M$-typical or not, admits a distribution of the values when the binary basic martingale difference sequence is such that $X_{i} \in\{-1,1\}$ for $i=1,2, X_{i} \in\{-2,2\}$ for $i=(2+1), \ldots,\left(2+2^{2}\right), X_{i} \in\{-1,1\}$ for $i=\left(2+2^{2}+1\right), \ldots,\left(2+2^{2}+2^{3}\right)$, and so forth. Note that, for univariate binary observations, with the specification of a basic martingale $M$ we get a structure which is essentially as rich as with the specification of a full probability distribution $\mathbf{P}$ over $\Omega^{\infty}$.

Let us start by considering the simple case of a univariate binary basic martingale difference sequence. Let $M_{n}=\sum_{i=1}^{n} X_{i}$ be a computable basic martingale such that $X_{i} \in\{a, b\}$, and $a\langle 0, b\rangle 0$ are rational numbers. For every $z \in \mathbf{R}$, we have that the indicator function $I_{(-\infty, z]}\left(X_{i}\right)=$ 
$(b-a)^{-1}\left(b-X_{i}\right), a \leqslant z<b$, it is equal to 0 , for $z<a$, and to 1 , for $z \geqslant b$, and so, that

$$
F_{n}(z)=\frac{1}{n} \sum_{i=1}^{n} I_{(-\infty, z]}\left(X_{i}\right)=\frac{b}{b-a}-\frac{1}{b-a} \frac{1}{n} \sum_{i=1}^{n} X_{i}, \quad a \leqslant z<b
$$

$F_{n}(z)=0, z<a$, and $F_{n}(z)=1, z \geqslant b$. Then, since any $M$-typical sequence $\xi$ is also $N$-typical, where $N$ is the computable stochastic sequence defined by

$$
N_{n}=\left[\sum_{i=1}^{n} X_{i},(a+b) \sum_{i=1}^{n} X_{i}\right]^{\prime}=\left[\sum_{i=1}^{n} X_{i}, \sum_{i=1}^{n}\left(X_{i}^{2}+a b\right)\right]^{\prime},
$$

we have, by an application of the SLLN of [5, Theorem 2]) taking $N$ as the basic martingale, that, for any $M$-typical sequence $\xi$,

$$
F_{n}(z)\left(\xi^{n}\right) \rightarrow \frac{b}{b-a}, \quad a \leqslant z<b, \text { as } n \rightarrow \infty .
$$

We will next examine the case in which $a=-1$ and $b=1$.

2.1. A symmetric Bernoulli stochastic sequence. Let us consider the symmetric Bernoulli stochastic sequence given by the computable basic martingale $M_{n}=\sum_{i=1}^{n} X_{i}$, with $X_{i} \in\{-1,1\}$. Let $\xi$ be an $M$-typical sequence, $N \gg q, N \in \mathbf{N}, q \in \mathbf{N}$, and consider the following auxiliary finite probability space. Define the $2^{q+1}+1$ sets

$$
\begin{aligned}
E_{--\cdots+} & =\left\{n: n \leqslant N, X_{n}=-1, X_{n-1}=-1, \ldots, X_{n-q}=-1\right\}, \\
E_{-\ldots+} & =\left\{n: n \leqslant N, X_{n}=-1, X_{n-1}=-1, \ldots, X_{n-q}=+1\right\}, \\
& \vdots \\
E_{++\cdots+} & =\left\{n: n \leqslant N, X_{n}=+1, X_{n-1}=+1, \ldots, X_{n-q}=+1\right\}, \\
E_{0} & =\left\{n: n \leqslant N, X_{m}=0 \text { for some } m=n, n-1, \ldots, n-q\right\},
\end{aligned}
$$

where $X_{0}=X_{-1}=\cdots=X_{-q}=0$. These sets are disjoint, their union is equal to the set $\{1,2, \ldots, N\}$ of the first $N$ natural numbers, and some of them could well be empty. We regard these sets formally. They are, empty or not, the «points» of a finite space $\Lambda_{q+1}$. On this space we consider the algebra $\mathscr{A}_{q+1}$ generated by the above sets, and the measure $\mu$ giving equal probability $1 / 2^{q+1}$ to all points $E \in \Lambda_{q+1}$, but to $E_{0}$ to which it gives probability zero.

On the probability space $\left(\Lambda_{q+1}, \mathscr{A}_{q+1}\right)$, define also the frequency measure $\nu_{N}$ given by

$$
\nu_{N}\left(E_{--\cdots-}\right)=\frac{1}{N} \#\left\{n: n \leqslant N, X_{n}=-1, X_{n-1}=-1, \ldots, X_{n-q}=-1\right\}
$$




$$
\begin{aligned}
\nu_{N}\left(E_{--\ldots+}\right) & =\frac{1}{N} \#\left\{n: n \leqslant N, X_{n}=-1, X_{n-1}=-1, \ldots, X_{n-q}=+1\right\}, \\
& \vdots \\
\nu_{N}\left(E_{++\cdots+}\right) & =\frac{1}{N} \#\left\{n: n \leqslant N, X_{n}=+1, X_{n-1}=+1, \ldots, X_{n-q}=+1\right\}, \\
\nu_{N}\left(E_{0}\right) & =\frac{1}{N} \#\left\{n: n \leqslant N, X_{m}=0 \text { for some } m=n, n-1, \ldots, n-q\right\} .
\end{aligned}
$$

Lemma 1. Let $M$ be the symmetric Bernoulli stochastic sequence. Then, for any $M$-typical sequence $\xi$, the measures $\nu_{N}$ tend to the measure $\mu$, uniformly over $\mathscr{A}_{q+1}, q \in \mathbf{N}$, as $N \rightarrow \infty$.

$\mathrm{P}$ r o o f. To show this, we will consider in turn the sets $E_{-}, E_{+}$, then the sets $E_{--}, E_{-+}, E_{+-}, E_{++}$, and so forth up to the sets $E \in \Lambda_{q+1}$. We start with the sets $E_{-}$and $E_{+}$. Let $\xi$ be an $M$-typical sequence. Since $X_{n} \in\{-1,1\}$, we have that $\sum_{n=1}^{N} X_{n}=\#\left\{n: n \leqslant N, X_{n}=1\right\}-\#\{n: n \leqslant$ $\left.N, X_{n}=-1\right\}$. So, since we can write

$$
\nu_{N}\left(E_{+}\right)=\frac{1}{N} \#\left\{n: n \leqslant N, X_{n}=1\right\}=\frac{1}{2}+\frac{1}{N} \sum_{n=1}^{N} \frac{1}{2} X_{n},
$$

$\nu_{N}\left(E_{-}\right)=1-\nu_{N}\left(E_{+}\right)$, by the SLLN of [5, Theorem 2]) we have that, for the $M$-typical sequence $\xi, \nu_{N}\left(E_{+}\right) \rightarrow \frac{1}{2}$ and $\nu_{N}\left(E_{-}\right) \rightarrow \frac{1}{2}$, as $N \rightarrow \infty$.

Let us consider the sets $E_{++}, E_{-+}, E_{+-}$and $E_{--}$. We define the computable predictable sequences

$$
V_{n}^{+}=\left\{\begin{array}{ll}
1, & X_{n-1}=1, \\
0, & \text { otherwise },
\end{array} \quad V_{n}^{-}= \begin{cases}1, & X_{n-1}=-1, \\
0, & \text { otherwise }\end{cases}\right.
$$

By using the predictable sequence $V_{n}^{+}$, similarly as before, we have that

$$
\begin{aligned}
\frac{1}{N} \sum_{n=1}^{N} V_{n}^{+} X_{n}= & \frac{1}{N}\left(\#\left\{n: n \leqslant N, X_{n}=1, X_{n-1}=1\right\}\right. \\
& \left.-\#\left\{n: n \leqslant N, X_{n}=-1, X_{n-1}=1\right\}\right),
\end{aligned}
$$

and so

$$
\begin{aligned}
\nu_{N}\left(E_{++}\right) & =\frac{1}{N} \#\left\{n: n \leqslant N, X_{n}=1, X_{n-1}=1\right\} \\
& =\frac{1}{4}+O\left(\frac{1}{N}\right)+\frac{1}{N} \sum_{n=1}^{N}\left(\frac{1}{2} V_{n}^{+}+\frac{1}{4}\right) X_{n}, \\
\nu_{N}\left(E_{-+}\right) & =\frac{1}{N} \#\left\{n: n \leqslant N, X_{n}=-1, X_{n-1}=1\right\} \\
& =\frac{1}{4}+O\left(\frac{1}{N}\right)+\frac{1}{N} \sum_{n=1}^{N}\left(-\frac{1}{2} V_{n}^{+}+\frac{1}{4}\right) X_{n} .
\end{aligned}
$$


Also, by considering the predictable sequence $V_{n}^{-}$, similar expressions can be written for $\nu_{N}\left(E_{+-}\right)$and $\nu_{N}\left(E_{--}\right)$, and by the variant of the SLLN for martingale transforms of [5] we have that, for the $M$-typical sequence $\xi, \nu_{N}\left(E_{++}\right) \rightarrow \frac{1}{4}, \nu_{N}\left(E_{-+}\right) \rightarrow \frac{1}{4}, \nu_{N}\left(E_{+-}\right) \rightarrow \frac{1}{4}$ and $\nu_{N}\left(E_{--}\right) \rightarrow \frac{1}{4}$, as $N \rightarrow \infty$.

For the sets $E_{+++}, E_{++-}, E_{+-+}, E_{+--}, E_{-++}, E_{-+-}, E_{--+}, E_{---}$, by using the computable predictable sequences $V_{n}^{++}, V_{n}^{+-}, V_{n}^{-+}, V_{n}^{--}$, defined by

$$
\begin{aligned}
& V_{n}^{++}=\left\{\begin{array}{ll}
1, & X_{n-1}=1, \\
0, & \text { otherwise, }
\end{array} \quad X_{n-2}=1,\right. \\
& V_{n}^{+-}=\left\{\begin{array}{ll}
1, & X_{n-1}=1, \\
0, & \text { otherwise, }
\end{array} \quad X_{n-2}=-1, \quad\right. \text { etc. }
\end{aligned}
$$

we have, for instance, that

$$
\nu_{N}\left(E_{+++}\right)=\frac{1}{8}+O\left(\frac{1}{N}\right)+\frac{1}{N} \sum_{n=1}^{N}\left(\frac{1}{2} V_{n}^{++}+\frac{1}{4} V_{n}^{+}+\frac{1}{8}\right) X_{n},
$$

and so, for the $M$-typical sequence $\xi, \nu_{N}\left(E_{+++}\right) \rightarrow \frac{1}{8}$, as $N \rightarrow \infty$. And similarly for the other sets. Proceeding in this way, we can show that, for the $M$-typical sequence $\xi$, for any set $E \in \Lambda_{q+1}$, but for $E_{0}$ for which $\nu_{N}\left(E_{0}\right) \rightarrow 0$, as $N \rightarrow \infty, \nu_{N}(E) \rightarrow 1 / 2^{q+1}$, as $N \rightarrow \infty$. Also, since $\Lambda_{q+1}$ is finite, $\nu_{N}(A) \rightarrow \mu(A)$, as $N \rightarrow \infty$, uniformly for all sets $A$ in the algebra $\mathscr{A}_{q+1}$, and thus, the result is proved.

2.2. A moving average stochastic sequence. Let us consider now a simple moving average stochastic sequence. Let $M_{n}=\sum_{i=1}^{n} X_{i}$ be the symmetric Bernoulli stochastic sequence, and consider the computable stochastic sequence defined by

$$
Y_{n}=X_{n}+\beta_{1} X_{n-1}+\beta_{2} X_{n-2}+\cdots+\beta_{q} X_{n-q}, \quad n=1,2, \ldots,
$$

where $X_{0}=X_{-1}=\cdots=X_{-q}=0$, and $\beta_{1}, \beta_{2}, \ldots, \beta_{q}$ are rational numbers.

Theorem 1. For the moving average stochastic sequence (1), for any $M$-typical sequence $\xi$, as $N \rightarrow \infty$,

$$
\frac{1}{N} \#\left\{n: n \leqslant N, Y\left(\xi^{n}\right) \leqslant z\right\} \longrightarrow \varphi(z),
$$

uniformly in $z \in \mathbf{R}$, where $\varphi(z)=\mathbf{P}\left\{Z_{0}+Z_{1}+\cdots+Z_{q} \leqslant z\right\}$, and $Z_{0}, Z_{1}, \ldots, Z_{q}$ are independent discrete random variables with $\mathbf{P}\left\{Z_{j}=\beta_{j}\right\}=$ $\mathbf{P}\left\{Z_{j}=-\beta_{j}\right\}=\frac{1}{2}, \beta_{0}=1, j=0,1, \ldots, q$. 
P r o o f. Consider $\left(\Lambda_{q+1}, \mathscr{A}_{q+1}\right), \mu$ and $\nu_{N}$ as defined before. On the probability space $\left(\Lambda_{q+1}, \mathscr{A}_{q+1}\right)$, define the random variables $Z_{0}, Z_{1}, \ldots, Z_{q}$ given by

$$
Z_{0}(E)=\left\{\begin{array}{rl}
1, & E=E_{+}, \\
0, & E=E_{0}, \\
-1, & E=E_{-} \ldots,
\end{array} \quad \ldots, \quad Z_{q}(E)=\left\{\begin{aligned}
\beta_{q}, & E=E \ldots+ \\
0, & E=E_{0} \\
-\beta_{q}, & E=E \ldots \ldots
\end{aligned}\right.\right.
$$

where $E \in \Lambda_{q+1}$. With respect to the measure $\mu$, these random variables are independent and such that $\mu\left(Z_{j}=\beta_{j}\right)=\mu\left(Z_{j}=-\beta_{j}\right)=\frac{1}{2}, j=0,1, \ldots, q$.

Then since, for every $z \in \mathbf{R}$, we have that

$$
\begin{aligned}
\frac{1}{N} & \#\left\{n: n \leqslant N, Y\left(\xi^{n}\right) \leqslant z\right\} \\
& =\frac{1}{N} \#\left\{n: n \leqslant N, n \in \text { some } E \text { such that } \sum_{j=0}^{q} Z_{j}(E) \leqslant z\right\} \\
& =\nu_{N}\left\{E: E \in \Lambda_{q+1}, \sum_{j=0}^{q} Z_{j}(E) \leqslant z\right\}=\nu_{N}\left(\sum_{j=0}^{q} Z_{j}(E) \leqslant z\right),
\end{aligned}
$$

the desired result is proved since by Lemma 1 the measures $\nu_{N}$ tend to the measure $\mu$, as $N \rightarrow \infty$, uniformly over $\mathscr{A}_{q+1}$.

2.3. A first-order autoregressive stochastic sequence. Let $M_{n}=$ $\sum_{i=1}^{n} X_{i}$ be the symmetric Bernoulli stochastic sequence, and consider the computable stochastic sequence defined by

$$
Y_{n}=\alpha Y_{n-1}+X_{n}, \quad n=1,2, \ldots,
$$

where $Y_{0}=0$, and $\alpha$, with $|\alpha|<1$, is a rational number.

Theorem 2. For the first-order autoregressive stochastic sequence (2), for any $M$-typical sequence $\xi$, as $N \rightarrow \infty$,

$$
\frac{1}{N} \#\left\{n: n \leqslant N, Y\left(\xi^{n}\right) \leqslant z\right\} \longrightarrow \varphi(z),
$$

uniformly in $z \in \mathbf{R}$, where $\varphi(z)=\lim _{r \rightarrow \infty} \varphi_{r}(z), \varphi_{r}(z)=\mathbf{P}\left\{Z_{1}+Z_{2}+\cdots+\right.$ $\left.Z_{r} \leqslant z\right\}$, and $Z_{1}, Z_{2}, \ldots, Z_{r}$ are independent discrete random variables with $\mathbf{P}\left\{Z_{j}=\alpha^{j-1}\right\}=\mathbf{P}\left\{Z_{j}=-\alpha^{j-1}\right\}=\frac{1}{2}, j=1,2, \ldots, r$.

P r o of. Let $\xi$ be an $M$-typical sequence, and consider, for large $N$, the computable stochastic sequences

$$
\begin{aligned}
& Z_{n}^{(1)}=X_{n}, \quad Z_{n}^{(2)}=\alpha X_{n-1}, \ldots, \\
& Z_{n}^{(j)}=\alpha^{j-1} X_{n-j+1}, \ldots, \quad Z_{n}^{(N)}=\alpha^{N-1} X_{n-N+1},
\end{aligned}
$$


where $X_{0}=0, X_{-1}=0, \ldots$ For any arbitrary $r, 1 \leqslant r \leqslant N$, we split $Y_{n}$ as follows,

$$
Y_{n}=\sum_{j=1}^{N} Z_{n}^{(j)}=\sum_{j=1}^{r} Z_{n}^{(j)}+\sum_{j=r+1}^{N} Z_{n}^{(j)}=Y_{n}^{\prime}(r)+Y_{n}^{\prime \prime}(r),
$$

say, and to show the theorem, by the properties of Lévy's metric, (see the remark following Lemma 1.7 in [2]) we just have to show that

$$
\begin{aligned}
& \text { (i) } \frac{1}{N} \#\left\{n: n \leqslant N, Y^{\prime}(r)\left(\xi^{n}\right) \leqslant z\right\} \rightarrow \varphi(z), \\
& \text { (ii) } \frac{1}{N} \#\left\{n: n \leqslant N,\left|Y^{\prime \prime}(r)\left(\xi^{n}\right)\right|>\varepsilon\right\} \longrightarrow 0, \text { for all } \varepsilon>0,
\end{aligned}
$$

for an $r=r(N) \rightarrow \infty$, as $N \rightarrow \infty$.

Let us show (i). For every fixed $r, Y_{n}^{\prime}(r)$ is a moving average stochastic sequence of order $q=r-1$, where $\beta_{j}=\alpha^{j}, j=0,1, \ldots, r-1$, and we have already seen that $(1 / N) \#\left\{n: n \leqslant N, Y^{\prime}(r)\left(\xi^{n}\right) \leqslant z\right\} \longrightarrow \varphi_{r}(z)$, uniformly in $z \in \mathbf{R}$, as $N \rightarrow \infty$. Then we can certainly find a sequence $\left\{\varepsilon_{r}\right\}, \varepsilon_{r}>0$, $\varepsilon_{r} \rightarrow 0$, as $r \rightarrow \infty$, and integers $\left\{N_{r}\right\}$, such that, for every $z \in \mathbf{R}$,

$$
\left|\frac{1}{N} \#\left\{n: n \leqslant N, Y^{\prime}(r)\left(\xi^{n}\right) \leqslant z\right\}-\varphi_{r}(z)\right|<\varepsilon_{r},
$$

for every $N \geqslant N_{r}$. Thus, there exists a function $r=r(N)$ which tends to infinity, as $N \rightarrow \infty$, slowly enough to ensure (i), uniformly in $z \in \mathbf{R}$, as $N \rightarrow \infty$.

Let us now show (ii). Note that $\left|Y_{n}^{\prime \prime}(r)\right|=0, n=1,2, \ldots, r$, and that

$$
Y_{n}^{\prime \prime}(r)=\sum_{i=1}^{n-r} \alpha^{n-i} X_{i}, \quad n=r+1, r+2, \ldots, N,
$$

that is, that

$$
\left|Y_{n}^{\prime \prime}(r)\right|=\left|\sum_{i=1}^{n-r} \alpha^{n-i} X_{i}\right| \leqslant \sum_{i=1}^{n-r} \alpha^{n-i}<\alpha^{r} \frac{1}{1-\alpha} .
$$

So, for every $\varepsilon>0$, there is an $r^{*}$ such that for every $r \geqslant r^{*},\left|Y_{n}^{\prime \prime}(r)\right|<\varepsilon$, and $(1 / N) \#\left\{n: n \leqslant N,\left|Y^{\prime \prime}(r)\left(\xi^{n}\right)\right|>\varepsilon\right\}=0$. That is, (ii) is true for any $r=r(N) \rightarrow \infty$, as $N \rightarrow \infty$. Theorem 2 is proved.

$\mathrm{R} \mathrm{e} \mathrm{m}$ a r $\mathrm{k} 1$. It might be noted that in the proof of Theorem 2 we used a technique, which relies on the properties of Lévy's metric, inspired by the proof of Theorem 5.2 of [2], [3], in which the limiting distribution of the values of additive arithmetic functions is studied. 


\section{The strong central limit theorem: preliminaries}

3.1. Shatte's results. Assuming Kolmogorov's axiomatics, let $X_{1}$, $X_{2}, \ldots$ be a sequence of independent identically distributed (i.i.d.) random variables, with $\mathbf{E}\left(X_{i}\right)=0, \mathbf{D}\left(X_{i}\right)=1$, and let $S_{n}=X_{1}+X_{2}+\cdots+X_{n}$. Then, by the classical central limit theorem, we know that the distribution of $S_{n} / \sqrt{n}$ converges to the standard normal distribution function $\Phi(z)$, as $n \rightarrow \infty$, but, of course, this result does not say anything about the distribution of the values of a single realization of the sequence of random variables $S_{n} / \sqrt{n}$. Indeed, Schatte [6] showed that, owing to the strong serial dependence in the sequence $\left(S_{n} / \sqrt{n}\right)$, the probability is zero that the arithmetic means of the sequence $I_{(-\infty, z]}\left(S_{n} / \sqrt{n}\right)$ converge, as $n \rightarrow \infty$, for all $z \in(-\infty, \infty)$. On the other hand, by considering «logarithmic means», Schatte proved the following result.

Theorem 3 ([6, Theorem 2]). Let $X_{1}, X_{2}, \ldots$ be i.i.d. random variables such that $\mathbf{E}\left(X_{i}\right)=0, \mathbf{D}\left(X_{i}\right)=1, \mathbf{E}\left(\left|X_{i}\right|^{3}\right)<\infty$, and $S_{n}=$ $X_{1}+X_{2}+\cdots+X_{n}$. Then

$$
\lim _{N \rightarrow \infty} \frac{1}{\ln N} \sum_{n=1}^{N} \frac{1}{n} I_{(-\infty, z]}\left(\frac{S_{n}}{\sqrt{n}}\right)=\Phi(z),
$$

almost surely, for all $z \in(-\infty, \infty)$.

Results of this kind, known as SCLT, were first shown independently by Schatte [6] and by Brosamler [1]. In addition, Schatte [6] showed that the dependence among the terms in the sequence $\left(S_{n} / \sqrt{n}\right)$ can also be overcome by considering a suitable thinning of the original sequence of values, proving the following SCLT for subsequences.

Theorem 4 ([6, Theorem 3]). Let $X_{1}, X_{2}, \ldots$ be i.i.d. random variables such that $\mathbf{E}\left(X_{i}\right)=0, \mathbf{D}\left(X_{i}\right)=1, \mathbf{E}\left(\left|X_{i}\right|^{3}\right)<\infty$, and $S_{n}$ be as before. Then for $n_{k}=\left\lfloor c^{k}\right\rfloor, c>1$, where $\left\lfloor c^{k}\right\rfloor$ is the integer part of $c^{k}$, we have that

$$
\lim _{K \rightarrow \infty} \frac{1}{K} \sum_{k=1}^{K} I_{(-\infty, z]}\left(\frac{S_{n_{k}}}{\sqrt{n_{k}}}\right)=\Phi(z),
$$

almost surely, for all $z \in(-\infty, \infty)$.

These early results have been extended, in the classical Kolmogorov probability axiomatics, and in a non-algorithmic framework, to a variety of other stochastic processes. These theorems, however, are usually proved by extending the SCLT for a sequence of i.i.d. random variables to the desired stochastic process, using some invariance principle, and a direct martingalebased proof of this result, in particular, does not yet seem available. On the other hand, in Section 4 we will prove some direct versions of the SCLT for $M$-typical sequences in the case of a univariate symmetric binary basic martingale. 
3.2. Preparation. Let us start by considering some properties about the indicator function of $M_{n} / \sqrt{n}$. Let us fix the univariate binary computable basic martingale $M_{n}=\sum_{i=1}^{n} X_{i}$, with $X_{i} \in\{-1,1\}$. Consider first $I_{(-\infty, z]}\left(M_{n} / \sqrt{n}\right)$ in the case $z=0$, for which it reduces to $I_{(-\infty, 0]}\left(M_{n}\right)$. For $n=1$, we simply have $I_{(-\infty, 0]}\left(X_{1}\right)=\frac{1}{2}-X_{1} / 2$. For $n=2$, by setting $I_{i}=I_{(-\infty, 0]}\left(X_{i}\right)$, and by considering all possible values of $X_{1}, X_{2}$, it is easy to check that

$I_{(-\infty, 0]}\left(X_{1}+X_{2}\right)=\left(1-I_{1}\right) I_{2}+I_{1}\left(1-I_{2}\right)+I_{1} I_{2}=\frac{3}{4}-\frac{X_{1}}{4}-\frac{X_{2}}{4}-\frac{X_{1} X_{2}}{4}$.

In the same way, we would also have, for $n=3$,

$$
\begin{gathered}
I_{(-\infty, 0]}\left(X_{1}+X_{2}+X_{3}\right)=I_{1} I_{2} I_{3}+I_{1} I_{2}\left(1-I_{3}\right)+I_{1}\left(1-I_{2}\right) I_{3} \\
+\left(1-I_{1}\right) I_{2} I_{3}=\frac{1}{2}-\frac{X_{1}}{4}-\frac{X_{2}}{4}-\frac{X_{3}}{4}+\frac{X_{1} X_{2} X_{3}}{4},
\end{gathered}
$$

and so forth, for all $n \in \mathbf{N}$. Here, just note that the sum of the coefficients of the expression in the $X_{i}$ is zero, for every $n \in \mathbf{N}$, since it is the value of the expression when all the $X_{i}$ are equal to one.

It is easy to see that all these indicator functions can be written as

$$
\begin{aligned}
& I_{(-\infty, 0]}\left(X_{1}\right)=\frac{1}{2}+V_{11} X_{1}, \\
& I_{(-\infty, 0]}\left(X_{1}+X_{2}\right)=\frac{3}{4}+V_{21} X_{1}+V_{22} X_{2}, \\
& I_{(-\infty, 0]}\left(X_{1}+X_{2}+X_{3}\right)=\frac{4}{8}+V_{31} X_{1}+V_{32} X_{2}+V_{33} X_{3}, \ldots,
\end{aligned}
$$

where the $V_{n i}$ depend only on $X_{1}, X_{2}, \ldots, X_{i-1}$, and that, noting that the predictable sequences defined in the proof of Lemma 1 satisfy

$$
\begin{aligned}
V_{n}^{-} & =I_{n-1}, \quad V_{n}^{+}=1-I_{n-1}, \\
V_{n}^{--} & =V_{n}^{-} V_{n-1}^{-}=I_{n-1} I_{n-2}, \quad V_{n}^{+-}=V_{n}^{+} V_{n-1}^{-}=\left(1-I_{n-1}\right) I_{n-2}, \quad \text { etc., }
\end{aligned}
$$

we can also write

$$
\begin{aligned}
& I_{(-\infty, 0]}\left(M_{1}\right)=\frac{1}{2}-\frac{1}{2} X_{1}, \\
& I_{(-\infty, 0]}\left(M_{2}\right)=\frac{3}{4}-\frac{1}{4} X_{1}-\frac{1}{2} V_{2}^{+} X_{2}, \\
& I_{(-\infty, 0]}\left(M_{3}\right)=\frac{4}{8}-\frac{1}{8} 2 X_{1}-\frac{1}{4} X_{2}-\frac{1}{2}\left(V_{3}^{+-}+V_{3}^{-+}\right) X_{3}, \ldots
\end{aligned}
$$

In this way, for every $n \in \mathbf{N}$, the indicator function of $M_{n}$, for $z=0$, can be written as a polynomial expansion in the $X_{i}$ of the form

$$
I_{(-\infty, 0]}\left(M_{n}\right)=\varphi_{n}+V_{n 1} X_{1}+\cdots+V_{n n} X_{n}
$$


where $\varphi_{n}$ is a rational number in $\left(\frac{1}{2}, 1\right]$, such that $\varphi_{n} \rightarrow \frac{1}{2}$, as $n \rightarrow \infty$, and the $V_{n i}$ are computable quantities which depend only on $X_{1}, X_{2}, \ldots, X_{i-1}$.

Consider now the case of a general $z \in \mathbf{R}_{c}$. Similarly to before, for every $n \in \mathbf{N}$, the indicator function $I_{(-\infty, z]}\left(M_{n} / \sqrt{n}\right)$ can be written as the sum of $m$ products of $n$ terms, where $m$ is the number of paths of length $n$. such that $M_{n} \leqslant \sqrt{n} z$, and the $n$ terms are of the form $\left(\frac{1}{2} \pm X_{i} / 2\right)$. That is, we can write

$$
I_{(-\infty, z]}\left(\frac{M_{n}}{\sqrt{n}}\right)=\varphi_{n}(z)+V_{n 1}(z) X_{1}+\cdots+V_{n n}(z) X_{n},
$$

where $\varphi_{n}(z)$ is a rational number which does not depend on the $X_{i}$, and the $V_{n i}(z)$ are computable quantities which depend on the $X_{i}$ only through $X_{1}, X_{2}, \ldots, X_{i-1}$.

By the structure of this polynomial expansion, it is easy to see that $\varphi_{n}(z)$ is just $m / n$, that is, the proportion of paths of length $n$ for which $M_{n} \leqslant \sqrt{n} z$. And, by introducing on $\Omega^{\infty}$ the auxiliary uniform Bernoulli measure $\mu$, since $\varphi_{n}(z)=\mu\left(M_{n} / \sqrt{n} \leqslant z\right)$, we also have that $\varphi_{n}(z) \rightarrow \Phi(z)$, as $n \rightarrow \infty$, by the De Moivre-Laplace central limit theorem [4, Theorem VII.3.2]. Let us note that here and later the measure $\mu$ plays only an auxiliary role and in our purely game-theoretic framework it does not have any probabilistic interpretation. Indeed, it just provides a convenient way to express an underlying combinatorial argument.

With the help of the auxiliary measure $\mu$, the quantities $V_{n i}(z)$ can be evaluated by considering the proportion of paths of length $n$ for which $M_{n} \leqslant \sqrt{n} z$, given the initial realizations $\left(X_{1}, X_{2}, \ldots, X_{i}\right)$. In fact, we have

$$
\begin{aligned}
& \mu\left(\frac{M_{n}}{\sqrt{n}} \leqslant z \mid X_{1}, \ldots, X_{i}\right)=\mu\left(I_{(-\infty, z]}\left(\frac{M_{n}}{\sqrt{n}}\right)=1 \mid X_{1}, \ldots, X_{i}\right) \\
& \quad=\mathbf{E}\left(I_{(-\infty, z]}\left(\frac{M_{n}}{\sqrt{n}}\right) \mid X_{1}, \ldots, X_{i}\right)=\varphi_{n}(z)+V_{n 1}(z) X_{1}+\cdots+V_{n i}(z) X_{i},
\end{aligned}
$$

where the expectation $\mathbf{E}(\cdot)$ is taken with respect to $\mu$, since, under $\mu$, the $X_{i}$ are independent random variables with $\mathbf{E}\left(X_{i}\right)=0$ and $\mathbf{D}\left(X_{i}\right)=1$. And so, by considering the two conditioning sets $\left(X_{1}, \ldots, X_{i-1}\right)$ and $\left(X_{1}, \ldots, X_{i-1}, X_{i}=1\right)$, we immediately have

$$
V_{n i}(z)=-\mu\left(\frac{M_{n}}{\sqrt{n}} \leqslant z \mid X_{1}, \ldots, X_{i-1}\right)+\mu\left(\frac{M_{n}}{\sqrt{n}} \leqslant z \mid X_{1}, \ldots, X_{i-1}, X_{i}=1\right) .
$$

By rewriting this last expression as

$$
V_{n i}(z)=-\mu\left(M_{n-i+1} \leqslant z^{*}\right)+\mu\left(M_{n-i} \leqslant z^{*}-1\right),
$$

where $z^{*}=z \sqrt{n}-\left(X_{1}+\cdots+X_{i-1}\right)$, it is possible to see that we can bound $V_{n i}(z)$ by $-2^{-1} \mu\left(M_{n-i}=0\right)$, or $-2^{-1} \mu\left(M_{n-i}=1\right)$, depending on whether 
$(n-i)$ is even or odd, which, by using Stirling's formula (see [4, Section II.9]), can both be approximated by

$$
-\frac{1}{\sqrt{2 \pi(n-i)}}, \quad \text { as } n \rightarrow \infty
$$

\section{The strong central limit theorem}

4.1. Logarithmic averages. The following result is a direct version for $M$-typical sequences of the SCLT for logarithmic averages. Note that, for finite $N$, the logarithmic average considered by Schatte's SCLT in (3) is not a distribution function, being greater than one for $z$ large enough. However, it tends to a distribution function, as $N \rightarrow \infty$.

Theorem 5. Consider the computable basic martingale $M_{n}=\sum_{i=1}^{n} X_{i}$, with $X_{i} \in\{-1,1\}$. Then, for any $M$-typical sequence $\xi$,

$$
\lim _{N \rightarrow \infty} \frac{1}{\ln N} \sum_{n=1}^{N} \frac{1}{n} I_{(-\infty, z]}\left(\frac{M_{n}}{\sqrt{n}}\right)=\Phi(z),
$$

uniformly in $z \in \mathbf{R}$, where $\Phi(z)$ is the standard normal distribution function.

P r o of. Let us fix a $z \in \mathbf{R}_{c}$. Since, from Section 3, for every $n \in \mathbf{N}$,

$$
I_{(-\infty, z]}\left(\frac{M_{n}}{\sqrt{n}}\right)=\varphi_{n}+V_{n 1} X_{1}+\cdots+V_{n n} X_{n},
$$

where $\varphi_{n}$ is a rational number in $[0,1]$, such that $\varphi_{n} \rightarrow \Phi(z)$, as $n \rightarrow \infty$, and the $V_{n i}$ are computable quantities which depend on the $X_{i}$ only through $X_{1}, X_{2}, \ldots, X_{i-1}$, we can write

$$
\begin{aligned}
& \frac{1}{\ln N} \sum_{n=1}^{N} \frac{1}{n} I_{(-\infty, z]}\left(\frac{M_{n}}{\sqrt{n}}\right) \\
& =\frac{1}{\ln N} \sum_{n=1}^{N} \frac{1}{n} \varphi_{n}+\frac{1}{\ln N} \sum_{n=1}^{N} \frac{1}{n}\left(V_{n 1} X_{1}+\cdots+V_{n n} X_{n}\right) .
\end{aligned}
$$

Since the first term on the right-hand side tends to $\Phi(z)$, as $N \rightarrow \infty$, it remains to show that the second term tends to zero, as $N \rightarrow \infty$. This can be written as

$$
\frac{1}{\ln N} R_{N}-\frac{1}{\ln N} \sum_{n=N+1}^{\infty} \frac{1}{n}\left(V_{n 1} X_{1}+\cdots+V_{n N} X_{N}\right),
$$

where

$$
\begin{aligned}
R_{N} & =\left(\sum_{r=1}^{\infty} \frac{1}{r} V_{r 1}\right) X_{1}+\left(\sum_{r=2}^{\infty} \frac{1}{r} V_{r 2}\right) X_{2}+\cdots+\left(\sum_{r=N}^{\infty} \frac{1}{r} V_{r N}\right) X_{N} \\
& =\sum_{i=1}^{N} W_{i} X_{i},
\end{aligned}
$$


say, is a computable $M$-martingale, and $W$ is a computable predictable sequence, negative, and, by using the asymptotic approximation (4), with

$$
\left|W_{i}\right|=\left|\sum_{r=i}^{\infty} \frac{1}{r} V_{r i}\right| \sim \frac{1}{\sqrt{2 \pi}} \sum_{r=1}^{\infty} \frac{1}{i+r} \frac{1}{\sqrt{r}} \leqslant \frac{c}{\sqrt{i}},
$$

where $c$ is a constant.

Then since, for $i_{j}=\left\lfloor e^{j}\right\rfloor$,

$$
\lim _{N \rightarrow \infty} \frac{1}{\ln N} \sum_{i=1}^{N} W_{i} X_{i}=\lim _{k \rightarrow \infty} \frac{1}{k} \sum_{j=1}^{k}\left(W_{i_{j-1}+1} X_{i_{j-1}+1}+\cdots+W_{i_{j}} X_{i_{j}}\right),
$$

and since

$$
\begin{aligned}
\sum_{j=1}^{\infty} \frac{1}{j^{2}}\left(W_{i_{j-1}+1}^{2}+\cdots+W_{i_{j}}^{2}\right) & <\sum_{j=1}^{\infty} \frac{1}{j^{2}}\left(\frac{c^{2}}{i_{j-1}+1}+\cdots+\frac{c^{2}}{i_{j}}\right) \\
& <\sum_{j=1}^{\infty} \frac{c^{2}}{j^{2}} \frac{i_{j}-i_{j-1}}{i_{j-1}+1}<\infty
\end{aligned}
$$

by the variant of the SLLN for sampled martingale transforms of [5] we have that, for any $M$-typical sequence, $(1 / \ln N) \sum_{i=1}^{N} W_{i} X_{i} \longrightarrow 0$, as $N \rightarrow \infty$. Thus, since by the upper half of the LIL of [5, Theorem 3], for any $M$-typical sequence,

$$
\lim _{N \rightarrow \infty} \frac{1}{\ln N}\left|M_{N}\right|\left|\sum_{n=N+1}^{\infty} \frac{1}{n} V_{n N}\right| \leqslant \lim _{N \rightarrow \infty} \frac{1}{\ln N} \sqrt{2 N \ln \ln N} \frac{c}{\sqrt{N}}=0
$$

and since the $V_{n i}$ are all non-positive, and for $i<N$,

$$
\left|\sum_{n=N+1}^{\infty} \frac{1}{n} V_{n i}\right|<\left|\sum_{n=N+1}^{\infty} \frac{1}{n} V_{n N}\right|
$$

where the term on the right-hand side tends to zero, as $N \rightarrow \infty$, we have that

$$
\begin{aligned}
& \lim _{N \rightarrow \infty} \frac{1}{\ln N} \sum_{n=N+1}^{\infty} \frac{1}{n}\left(V_{n 1} X_{1}+\cdots+V_{n N} X_{N}\right) \\
& =\lim _{N \rightarrow \infty} \frac{1}{\ln N} \sum_{n=N+1}^{\infty} \frac{1}{n}\left(V_{n N} X_{1}+\cdots+V_{n N} X_{N}\right)=0
\end{aligned}
$$

and the theorem is proved.

The next result is about the first moment, in logarithmic average, of the standardized martingale $M_{n} / \sqrt{n}$. 
Lemma 2. Consider the computable basic martingale $M_{n}=\sum_{i=1}^{n} X_{i}$, with $X_{i} \in\{-1,1\}$. Then, for any $M$-typical sequence $\xi$,

$$
\lim _{N \rightarrow \infty} \frac{1}{\ln N} \sum_{n=1}^{N} \frac{1}{n} \frac{M_{n}}{\sqrt{n}}=0 .
$$

P r o of. Write

$$
\frac{1}{\ln N} \sum_{n=1}^{N} \frac{1}{n} \frac{M_{n}}{\sqrt{n}}=\frac{1}{\ln N} \sum_{i=1}^{N} W_{i} X_{i}-\frac{1}{\ln N} \sum_{n=N+1}^{\infty} \frac{1}{n} \frac{M_{n}}{\sqrt{n}},
$$

where $W_{i}=\sum_{r=i}^{\infty} r^{-3 / 2}=o\left(i^{-1 / 2}\right)$, is a computable predictable sequence.

Then, by considering that $\sum_{i=1}^{N} W_{i} X_{i}$ is a computable $M$-martingale, since, for $i_{j}=\left\lfloor e^{j}\right\rfloor$,

$$
\lim _{N \rightarrow \infty} \frac{1}{\ln N} \sum_{i=1}^{N} W_{i} X_{i}=\lim _{k \rightarrow \infty} \frac{1}{k} \sum_{j=1}^{k}\left(W_{i_{j-1}+1} X_{i_{j-1}+1}+\cdots+W_{i_{j}} X_{i_{j}}\right),
$$

and since

$$
\begin{aligned}
\sum_{j=1}^{\infty} \frac{1}{j^{2}}\left(W_{i_{j-1}+1}^{2}+\cdots+W_{i_{j}}^{2}\right) & <\sum_{j=1}^{\infty} \frac{1}{j^{2}}\left(\frac{c^{2}}{i_{j-1}+1}+\cdots+\frac{c^{2}}{i_{j}}\right) \\
& <\sum_{j=1}^{\infty} \frac{c^{2}}{j^{2}} \frac{i_{j}-i_{j-1}}{i_{j-1}+1}<\infty,
\end{aligned}
$$

by the variant of the SLLN for sampled martingale transforms of [5] we have that, for any $M$-typical sequence, $(1 / \ln N) \sum_{i=1}^{N} W_{i} X_{i} \longrightarrow 0$, as $N \rightarrow \infty$.

Moreover, by using the upper half of the LIL of [5, Theorem 3], we have that, for any $M$-typical sequence,

$$
\lim _{N \rightarrow \infty}\left|\frac{1}{\ln N} \sum_{n=N+1}^{\infty} \frac{1}{n} \frac{M_{N}}{\sqrt{n}}\right| \leqslant \lim _{N \rightarrow \infty} \frac{1}{\ln N} \sqrt{2 N \ln \ln N} \frac{c}{\sqrt{N+1}}=0 .
$$

Lemma 2 is proved.

Let us note that we might have considered all the moments, in logarithmic average, of the standardized martingale $M_{n} / \sqrt{n}$ in a similar way.

4.2. Subsequences. The following theorem is a direct version for $M$-typical sequences of Schatte's SCLT for subsequences. It is worth noting that its proof follows closely the proof of Theorem 5. Let us first consider the following definition. For every fixed rational number $\alpha \in(0,1)$, let $\left\{n_{k}\right\}$ be the computable subsequence of $\{n\}$ such that $n_{0}=0, n_{1}=1$, and, for $k=2,3, \ldots, n_{k}$ is the first integer for which

$$
\frac{\sqrt{n_{k-1}}}{\sqrt{n_{k}}} \leqslant \alpha
$$


Theorem 6. Consider the computable basic martingale $M_{n}=\sum_{i=1}^{n} X_{i}$, with $X_{i} \in\{-1,1\}$, and, for a fixed rational number $\alpha \in(0,1)$, the computable subsequence $\left\{n_{k}\right\}$ given by (5). Then, for any $M$-typical sequence $\xi$,

$$
\lim _{K \rightarrow \infty} \frac{1}{K} \sum_{j=1}^{K} I_{(-\infty, z]}\left(\frac{M_{n_{j}}}{\sqrt{n_{j}}}\right)=\Phi(z),
$$

uniformly in $z \in \mathbf{R}$.

P r o of. Let us fix a $z \in \mathbf{R}_{c}$. From Section 3 , for every $n_{j}, j=1,2, \ldots$,

$$
I_{(-\infty, z]}\left(\frac{M_{n_{j}}}{\sqrt{n_{j}}}\right)=\varphi_{n_{j}}+V_{n_{j}, 1} X_{1}+\cdots+V_{n_{j}, n_{j}} X_{n_{j}},
$$

where $\varphi_{n_{j}}$ is a rational number in $[0,1]$, such that $\varphi_{n_{j}} \rightarrow \Phi(z)$, as $n_{j} \rightarrow \infty$, and the $V_{n_{j}, i}$ are computable quantities which depend on the $X_{i}$ only through $X_{1}, X_{2}, \ldots, X_{i-1}$. Then we have

$$
\frac{1}{K} \sum_{j=1}^{K} I_{(-\infty, z]}\left(\frac{M_{n_{j}}}{\sqrt{n_{j}}}\right)=\frac{1}{K} \sum_{j=1}^{K} \varphi_{n_{j}}+\frac{1}{K} \sum_{j=1}^{K}\left(V_{n_{j}, 1} X_{1}+\cdots+V_{n_{j}, n_{j}} X_{n_{j}}\right)
$$

and since the first term on the right-hand side tends to $\Phi(z)$, as $K \rightarrow \infty$, we have to show that the second term tends to zero, as $K \rightarrow \infty$. To show this, write it as

$$
\frac{1}{K} R_{K}-\frac{1}{K} \sum_{j=K+1}^{\infty}\left(V_{n_{j}, 1} X_{1}+\cdots+V_{n_{j}, n_{K}} X_{n_{K}}\right)
$$

where

$$
R_{K}=\sum_{j=1}^{K}\left(W_{n_{j-1}+1} X_{n_{j-1}+1}+\cdots+W_{n_{j}} X_{n_{j}}\right)
$$

and $W_{i}=\sum_{r=j}^{\infty} V_{n_{r}, i}, n_{j-1}<i \leqslant n_{j}$, is a negative computable predictable sequence, such that, by using the asymptotic approximation (4),

$$
\begin{aligned}
\left|W_{n_{j}}\right| & =\left|\sum_{r=j}^{\infty} V_{n_{r}, n_{j}}\right| \sim \frac{1}{\sqrt{2 \pi}} \sum_{r=j+1}^{\infty} \frac{1}{\sqrt{n_{r}-n_{j}}} \\
& \leqslant \frac{1}{\sqrt{2 \pi}} \frac{1}{\sqrt{n_{j}}} \sum_{r=1}^{\infty} \frac{\alpha^{r}}{\sqrt{1-\alpha^{2 r}}} \leqslant c \alpha^{j-1}
\end{aligned}
$$

where $c$ is a constant.

Then, since $\left|W_{i}\right| \leqslant\left|W_{n_{j}}\right|$, when $i \leqslant n_{j}$,

$$
\begin{aligned}
\sum_{j=1}^{\infty} \frac{1}{j^{2}}\left(W_{n_{j-1}+1}^{2}+\cdots+W_{n_{j}}^{2}\right) & \leqslant \sum_{j=1}^{\infty} \frac{1}{j^{2}} W_{n_{j}}^{2}\left(n_{j}-n_{j-1}\right) \\
& \leqslant c^{2}\left(1-\alpha^{2}\right) \sum_{j=1}^{\infty} \frac{1}{j^{2}}<\infty
\end{aligned}
$$


and by the variant of the SLLN for sampled martingale transforms of [5] we have that, for any $M$-typical sequence, $\lim _{K \rightarrow \infty}(1 / K) R_{K}=0$. Also, by the upper half of the LIL of [5, Theorem 3], for any $M$-typical sequence,

$$
\lim _{K \rightarrow \infty} \frac{1}{K}\left|M_{n_{K}}\right|\left|\sum_{j=K+1}^{\infty} V_{n_{j}, n_{K}}\right| \leqslant \lim _{K \rightarrow \infty} \frac{1}{K} \sqrt{2 n_{K} \ln \ln n_{K}} \frac{c}{\sqrt{n_{K}}}=0
$$

and since the $V_{n_{j}, i}$ are all non-positive, and for $i<n_{K}$,

$$
\left|\sum_{j=K+1}^{\infty} V_{n_{j}, i}\right|<\left|\sum_{j=K+1}^{\infty} V_{n_{j}, n_{K}}\right|,
$$

where the term on the right-hand side tends to zero, as $K \rightarrow \infty$, we have that

$$
\begin{aligned}
& \lim _{K \rightarrow \infty} \frac{1}{K} \sum_{j=K+1}^{\infty}\left(V_{n_{j}, 1} X_{1}+\cdots+V_{n_{j}, n_{K}} X_{n_{K}}\right) \\
& =\lim _{K \rightarrow \infty} \frac{1}{K} \sum_{j=K+1}^{\infty}\left(V_{n_{j}, n_{K}} X_{1}+\cdots+V_{n_{j}, n_{K}} X_{n_{K}}\right)=0
\end{aligned}
$$

and the theorem is proved.

Let us finally consider the analogue for subsequences of Lemma 2, which, although presented as the last result in the series, can be seen as the prototype result of this section.

Lemma 3. Consider the computable basic martingale $M_{n}=\sum_{i=1}^{n} X_{i}$, with $X_{i} \in\{-1,1\}$, and, for a fixed rational number $\alpha \in(0,1)$, the computable subsequence $\left\{n_{k}\right\}$ given by (5). Then, for any $M$-typical sequence $\xi$,

$$
\lim _{K \rightarrow \infty} \frac{1}{K} \sum_{j=1}^{K} \frac{M_{n_{j}}}{\sqrt{n_{j}}}=0
$$

P r o of. We have

$$
\frac{1}{K} \sum_{j=1}^{K} \frac{M_{n_{j}}}{\sqrt{n_{j}}}=\frac{1}{K} \sum_{j=1}^{K} W_{j}\left(X_{n_{j-1}+1}+\cdots+X_{n_{j}}\right)-\frac{1}{K} \sum_{j=K+1}^{\infty} \frac{M_{n_{K}}}{\sqrt{n_{j}}},
$$

where

$$
W_{j}=\sum_{r=j}^{\infty} \frac{1}{\sqrt{n_{\tau}}} \leqslant \sum_{r=j}^{\infty} \alpha^{r-1}=\frac{\alpha^{j-1}}{1-\alpha}
$$

since $\sqrt{n_{j-1}} / \sqrt{n_{j}} \leqslant \alpha$. Then

$$
\sum_{j=1}^{\infty} \frac{1}{j^{2}} W_{j}^{2}\left(n_{j}-n_{j-1}\right) \simeq \frac{1-\alpha^{2}}{(1-\alpha)^{2}} \sum_{j=1}^{\infty} \frac{1}{j^{2}}<\infty,
$$


and by the variant of the SLLN for sampled martingale transforms in [5] we have that, for any $M$-typical sequence, $\lim _{K \rightarrow \infty}(1 / K) \sum_{j=1}^{K} W_{j}\left(X_{n_{j-1}+1}+\right.$ $\left.\cdots+X_{n_{j}}\right)=0$. Also, by the upper half of the LIL in [5, Theorem 3], for any $M$-typical sequence,

$$
\lim _{K \rightarrow \infty} \frac{1}{K}\left|M_{n_{K}}\right| \sum_{j=K+1}^{\infty} \frac{1}{\sqrt{n_{j}}} \leqslant \lim _{K \rightarrow \infty} \frac{1}{K} \sqrt{2 n_{K} \ln \ln n_{K}} \frac{\alpha^{K}}{1-\alpha}=0
$$

and the result is proved.

$\mathrm{R}$ e $\mathrm{m}$ a r k 2. It might be observed that in our proofs of the above SCLT type results the use of the SLLN is not sufficient, and we have to resort to the upper half of the LIL. As far as we know, Theorem 5 and 6 represent the first algorithmic versions, in whatever probability foundations, of the SCLT. In fact, it seems that in the traditional algorithmic probability frameworks, where a Kolmogorovian probability distribution $\mathbf{P}$ is employed, no version of this result has yet been proved for some random (typical or chaotic) sequences.

Acknowledgments. I would like to acknowledge that this research was carried out as part of the author Ph. D. at University College London and I express my gratitude to Professor A. Ph. Dawid and to Dr. V. G. Vovk. I am also grateful to the «Centro Nazionale delle Ricerche» for partial financial support.

\section{REFERENCES}

1. Brosamler G. An almost everywhere central limit theorem. - Math. Proc. Cambridge Philos. Soc., 1988, v. 104, p. 561-574.

2. Elliott P. D. T. A. Probabilistic Number Theory. I: Mean-Value Theorems. New York: Springer-Verlag, 1979, 393 p.

3. Elliott P.D.T.A. Probabilistic Number Theory. II: Central Limit Theorems. New York: Springer-Verlag, 1980, 375 p.

4. Феллер B. Введение в теорию вероятностей и ее приложения. Т. 1 . М.: Мир, $1984,528 \mathrm{c}$.

5. Minozzo $M$. Purely game-theoretic random sequences: I. Strong law of large numbers and law of the iterated logarithm. - Теория вероятн. и ее примен., 1999, т. 44, B. 3 , c. $617-630$.

6. Schatte $P$. On strong versions of the central limit theorem. - Math. Nachr., 1988, v. 137, p. $249-256$.

Поступила в редакцию 17.VII.1997

Переработанный вариант 11.XI.1998 